

\title{
RESEARCHING COLLABORATIVELY WITHIN IN-SERVICE CONTEXTS: SOME REFLECTIONS ON THE EXPERIENCES OF THE SEITT AT THE UNIVERSITY OF ZIMBABWE
}

\author{
David K.J. Mtetwa \\ Department of Science and Mathematics Education \\ University of Zimbabwe
}

\section{Abstract}

The first phase of the Science Education In-service Teucher Training (SEITT) from 1995 to 1998 was largely a construction phase, that is, putting into place appropriate physical, management, and opcrational structurcs for the programme. The second phase from 1999 to 2002 focused on consolidating the structures and operations created during the first phase. Although each phase contained a research element, the nature of the resench activity in the phases was somewhat different. The main outcomes accomplished by the research programme in the second phase of the SEITT were: enhancement of a sense of professionalism among the participants, development of research skills, establishment of socio-professional relátions (huddly partnerships), publication of articles contributing to the growth of knowledge in in-service education, and increased advocacy for the SEITT model of in-service education. The main difficulties experienced in the execution of the programme included those that had to do with allegiance to team membership, attrition. and logistics for meetings and writing. Two important observations can be made from a reflective analysis of this research programme. First, there is need for some form of recognition of active teacher researchers within the Ministry's reward system, and second, the science and mathematics centres could be turned into research centre sites for both conceptualising and conducting this kind of research and disseminating the findings. The SEITT team has also gained from this experience a number of important insights on collaborative research as a mode of professional development, especially, within in-service contexts. These include issues of team size and composition. inter-team member relations, and problems of ownership of research products that often brew acrimonious relations. 


\section{Introduction}

The practice of collaborative research appears to have caught on quite well in education faculties in some higher education institutions. In the Department of Science and Mathenatics Education at the University of Zimbabwe, we experienced it more recently within the framework of a continuing professional development (CPD) programme called SEITT.

The first phase of that programme ran from 1995 to 1998 and was largelya construction phase concentrating on creating and installing structures and designing operational processes. It was during this phase that science and mathematics centres (SMCs) for teachers, management committees, and linkage structures were established. New service and training programmes were also designed during this time. The second phase of SEITT ran from 1.999 to 2002 and focused on consolidating the structures and operations created during the first phase. This means that we had to ensure that materials (e.g., reference books and computing equipment) and activities (e.g., workshops, seminars, and planning meetings) that define the programme and the SMCs are in place and functioning properly. The issue of quality began to get increased attention during the second phase.

Both phases had a research element within them. However, the nature of the research activity was different in each case. The research activity in phase one largely consisted of feasibility and small formative evaluation studies by the SEITT team or their guests, and small scale action inquiry studies by trainee resource teachers as part of their research methods course. Most of the studies conducted during this phase can at best be described as 'quasi-research' studies in terms of the level of rigour. There was, however, one doctoral research study done by a guest of the SEITT team on the impact of the SEITT diploma-training programme on classroom practice.

The research element during phase two also had its own distinctive features. First, the research activity was organised and coordinated into a clear unified programme that was motivated, in part, by the desire by members of the university department to maintain professional relations and contacts with their graduates, and field test the new and exciting (in the Zimbabwean context) idea of collaborative research as a medium of cultivating such relations.

Second, the programme had more clearly articulated objectives that included

a informing in-service training in DSME and practice in the field 


\section{Zimbabwe Journal of Educational Research}

- developing à culture of research-based practice in the field, and

- developing a knowledge base in the domain of in-service education within the Zimbabwean context, and in that way, contribute towards the development of policy and theory on in-service education in Zimbabwe.

Third, the prime movers of the programme were supposed to be the university department (led by the SEITT team), teacher practitioners (represented by selected resource,teachers), Ministry of Education personnel (represented by interested education officers), with the department's technicians involved in a supporting role.

\section{Expected Outcomes}

- It was envisaged that through this coordinated research programme:

- graduate resource teachers will enhance their professional and career prospects;

- the authentic research would provide a good background for future engagements in higher education by participating resource teachers;

- some participating members from the university department could engage in research that could move them towards their doctoral qualifications;

- some publications could result from new home-grown knowledge in inservice education that would be generated;

- expertise in the field of CPD would be increased within the university, the ministry of education, and the schools;

- research skills of participants would be more horned, and;

- there would be increased advocacy for the SEITT model of CPD.

Beyond phase two, it was envisaged that a community of educational researchers and a viable mechanism for collaboration between the Ministry, the university, and the field would be created to last.

\section{Programme Design (Action Plan)}

First, we identified five research themes that related with the broad range of the SEITT dimensions of in-service activities. These were (a) networking, (b) 
science and mathematics centres, (c) instructional design and implementation, (d) materials development, and (e) curriculum development and implementation. Later, we added a sixth theme, laboratory equipment production. This was done to ensure that our research activity would remain integrated with all the other activities of the SEITT programme. This way research would continue to play the role of quality assurance within the programme.

Second, research teams (one for each theme) coordinated by a SEITT team member, were formed. The teams ranged in size from four to six persons in the initial stages to allow for attrition. Resource teacher participants were selected after assessing their interest in and capacity for research for a specific research activity, and balance in subject representation among mathematics, chemistry, biology, and physics.

Third, there were whole group, theme team, and individuál working sessions for developing the studies from proposal phase to paper writing. Both face-toface and electronic media were used for interactions among team members. Data gathering and writing tasks were mostly done at individual level (duties were allocated within theme teams) and proposal development and analysis was typically done during theme team sessions.

Fourth, the nature of expected outcomes (journal articles, progress reports, and conference paper presentations) ensured that high standards of rigour in both the conceptualisation and conduct of the research were enforced. To enhance that aspect, we incorporated whole group sessions for inter-theme team critiquing, and participation at local and regional SAARMSTE conferences for further scrutiny of our research processes and products.

Finally, an important feature of the programme was that team relations were supposed to reflect equality of researchers and not the asymmetry of a researcher and his/her assistants. That point was emphasised to members right from the beginning of the programme.

\section{Accomplishments}

Although not all the desired outcomes were accomplished, on the whole, the following aspects, were realised:

- enhancement of professionalism (many participants, especially the resource teachers, admitted that at the end they felt a sense of greater selfempowerment, autonomy, and career enhancement);

- research skills for all participants were enhanced (of the nearly 25 partici- 


\section{Zimbubwe Journal of Educational Research}

pants, two have enrolled in masters degree programmes, three in doctoral degree programmes, and a good number are seeking similar opportunities);

- widening of professional networks (most especially through exposure to the SAARMSTE forum, at both the local and the regional levels

- enhancement of writing skills (through the preparation of reports of the studies conducted for journal publications and conference proceedings. By the end of the programme over 10 papers would have been produced and published by the theme teams;

- establishment of socio-professional relations (this will appear as an obvious observation to the participants. A number of the partnerships are set to continue even after the SEITT/phase two period, and

increased levels of advocacy for the SEITT model and the strengthening of a research culture (many of the participants have increased their value for research and the notion of learning skills by doing).

\section{Difficulties}

The programme did experience some of the problems typical with teamwork such as attrition (due to death, translocation, surrender, loss of interest, etc), logistics of meeting, and disruptions due to member absences from meetings or member abrogation of duties. Administration of funding within the University of Zimbabwe in the final year of the project period became so stifling as to negatively impact on the pace and quality of the research programme. Finally, the problem of ownership of the process and product of the research surfacing on some occasions was experienced. The problem was reflected in some team members taking the orientation that their involvement was like an 8 am to 5pm affair, and in feeling like they were researching for somebody else (and therefore were being used) and not for themselves. This kind of problem is one that can only be resolved by the researcher involved. About one third of the team members were never able to resolve that problem in themselves right through to the end.

\section{Future Possibilities}

A reflective analysis of the programme:

confirms the somewhat obvious observation that research activity requires funding for it to happen. Án interesting consideration for us to 
think about is, who else could fund this kind of research programme other than a donor?

- makes it clear that there is need for active researchers in the school system to be properly recognised and acknowledged (promotion, status; money, talks, etc)

- suggests science and mathematics centres have the potential to develop into centres for both conducting and disseminating outcomes of this kind of research, and

- points to an important role SAARMSTE-Zimbabwe chapter can play in encouraging and strengthening collaborative research studies.

\section{Concluding Remarks}

I conclude by highlighting the following observations:

- The composition of collaborators is critical. Selection criteria should include an honest assessment of research potential profile and level of intrinsic motivation of the aspiring team member;

- There is need for researchers to integrate research with their own professional work

- Collegial orientation (mutual respect and equality among group members) is essential in this kind of enterprise. No sense of using each other should be allowed to develop;

- Collegial orientation, however, needs to be balanced by the need for the team leader (who ought to exist) to be both mentor/promoter for the team members. The team leader should be less of a teacher/lecturer for the group, though moments of teaching by the team leader may from time to time be instrumental for progress. Still, the team leader should work just like the other members.

- Discuss and agree on the first author responsibilities up-front, and as much as possible, rotate that responsibility.

- Team size of three to four is ideal. This maximises sharing of duties and perspectives, enhancing triangulation and at the same time minimises excess baggage carrying and group management and disruption problems (e.g., absenteeism);

- Finally, recognise team members' strengths and weaknesses and turn them into advantages for the team. 


\section{(c) (1) (9)}

This work is licensed under a

Creative Commons

Attribution - NonCommercial - NoDerivs 3.0 License.

To view a copy of the license please see:

http://creativecommons.ora/licenses/bv-nc-nd/3.0/ 